\title{
Editorial
}

\section{Procalcitonin (PCT) - The new era for diagnosis of infection and guiding antibiotic decisions}

There are a lot of limitations to use conventional diagnostic markers for patients with clinical suspicion of infection. As a consequence, unnecessary and prolonged exposure to antimicrobial agents adversely affects patient outcomes, while inappropriate antibiotic therapy increases antibiotic resistance. Emerging bacterial resistance to antimicrobial therapeutics calls for more efforts to reduce the overuse. Towards this aim, there has been considerable interest in antibiotic stewardship programs aimed at reducing antibiotic overuse by tailoring antibiotic therapy to individual needs of patients.

The disadvantages of many current microbiological methods are diagnostic delays (e.g. culture methods), suboptimal sensitivity (e.g. blood cultures) and low specificity due to contamination (e.g. sputum cultures). Others are not amenable to routine diagnostics due to their invasive nature (e.g. lung biopsy). Inflammatory markers, such as C-reactive protein or WBC count lack specificity for bacterial infections. In this diagnostic dilemma, Procalcitonin (PCT) has stimulated great interest as a potential more specific marker for bacterial infection. PCT is produced exclusively in response to endotoxin or mediators released in bacterial infections. They are interleukin (IL)-1 b, tumor necrosis factor (TNF), and IL-6. They strongly correlate with extent and severity of bacterial infections. Thus PCT is more specific for bacterial infections and may help to distinguish bacterial infections from viral illnesses. PCT shows a favorable kinetic profile for use as a clinical marker. It promptly increases within 6 to 12 hours upon stimulation and circulating PCT levels halve daily when the infection is controlled by the host immune system or antibiotic therapy. PCT correlates with bacterial load and severity of infection.

For infections like meningitis, severe sepsis, upper airway and or lower respiratory tract infection in ICU patients, ventilation associated pneumonia; postoperative infections and sepsis in critically ill patients randomized controlled studies have shown the efficacy of using PCT algorithms to guide antibiotic decisions.
For other types of infections like endocarditis, pyelonephritis/UTI, arthritis, intra-abdominal sepsis, bacteremia, only observational studies are available where evidences are limited by the lack of true gold standard. Another important issue is that PCT levels must always be evaluated in the context of a careful clinical and microbiological assessment. As the kinetics of PCT is of particular diagnostic and prognostic interest, repeated measurements should be performed if feasible, especially in persistently sick patients if antibiotics are withheld. Thus repeated measurements should be encouraged only in patients of ICU or High dependency unit (HDU) or Coronary care unit (CCU) or Neutropenia. Observing the cost effectiveness, PCT assessment is not suitable for outpatients or mild infections in stable patients.

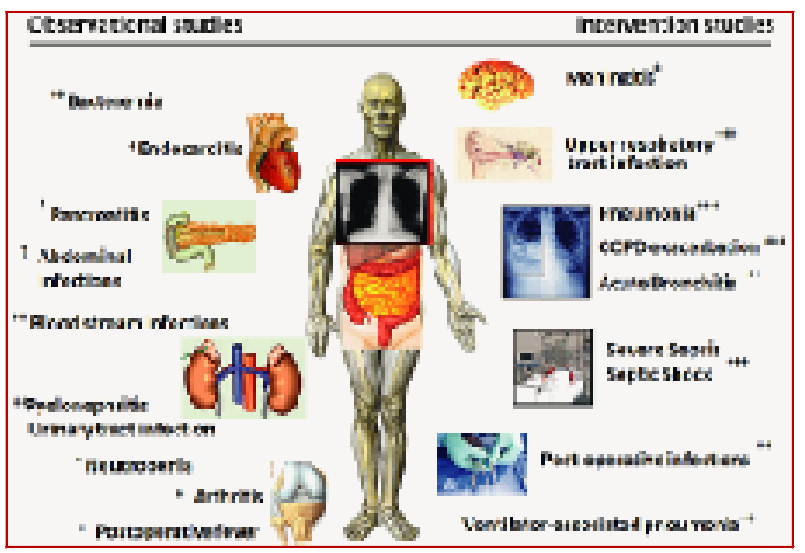

The limitations of every PCT measurement include false-positive and false-negative results. Unspecific elevations of PCT levels in the absence of a bacterial infection can typically be seen in situations of massive cell death, for example after severe trauma. In these situations, PCT values are usually only moderately elevated and show a rapid decline in follow-up measurements. Conversely, falsely low PCT levels, typically seen during the early course or localized state of an infection, may show an increase in the follow-up measurements. Therefore, highly sensitive PCT assays are required, as subtle changes of PCT at very low concentrations can be monitored, increasing the sensitivity and safety of patients. 
Emerging bacterial resistance to different antimicrobials calls for more effective efforts to reduce the unnecessary and prolonged use of antibiotics in self-limiting non bacterial and resolving diseases. Patients and physicians share a common goal of improving symptoms from infection as fast as possible and often see antibiotics as the most expeditious intervention to achieve it. This random approach fails to consider the basic questions of who benefits from antibiotic therapy, and if treated, what would be the optimal duration. Using PCT, which mirrors the likelihood of bacterial infection and the severity of infection, to guide antibiotic therapy, is a persuasive, evidence-based approach to a more rational use of antibiotics.

Source: Bio-med central (BMC) - Clinical Biomarkers

\section{Dr. Kazi Shihab Uddin MRCP (UK) Assistant Professor Department of Medicine $\mathrm{KYAMCH}$}

